\title{
Interface identification by non-local autoionization transitions
}

\section{Silko Barth, ${ }^{a}$ Simon Marburger, ${ }^{a}$ Sanjeev Joshi, ${ }^{a}$ Volker Ulrich, ${ }^{a}$ Oliver Kugeler ${ }^{a, b}$ and Uwe Hergenhahn ${ }^{a}$}

Receipt/Acceptance Data $10^{\text {th }}$ February 2006/ 30 ${ }^{\text {th }}$ May 2006

Publication data $9^{\text {th }}$ June 2006

5 Published in Physical Chemistry Chemical Physics 2006, 8, 3218-3222, DOI: 10.1039/b602019d

We use an autoionization process that involves ultrafast energy transfer to neighbouring sites to characterize the formation of NeAr van der Waals bonds in clusters formed by a coexpansion of both gases. This autoionization process, the so-called Interatomic or Intermolecular Coulombic Decay (ICD), is ubiquitous in weakly bonded systems. The energy of the electron being emitted in ${ }_{10}$ the ICD process is shown to be characteristic for the two neighbouring entities and is therefore suggested as a new means for structural investigation, such as interface identification, of weakly bonded complexes.

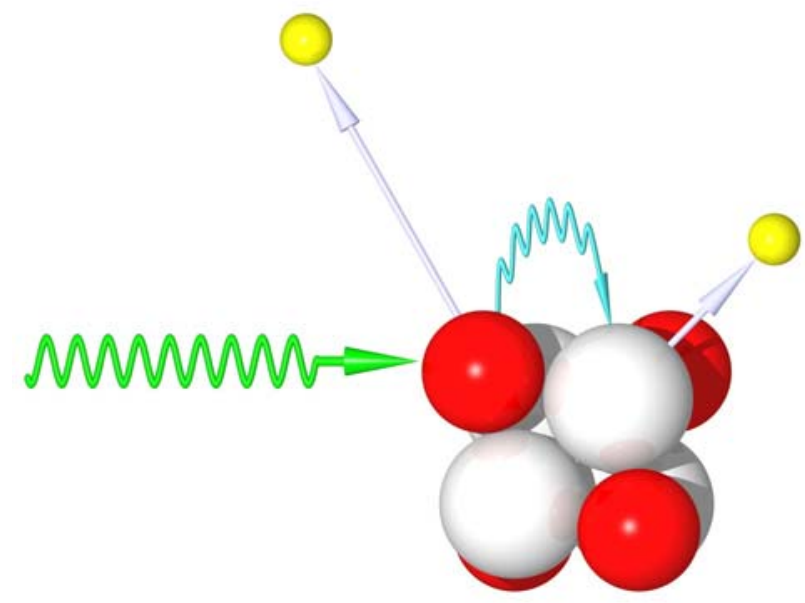

\section{${ }_{15}$ Introduction}

Non-covalently bonded species are of great importance in chemistry and biochemistry. Since non-covalent bonds, e.g. via hydrogen bridge bonds or van der Waals interactions, are weak, the properties of the subsystems are relatively 20 unperturbed compared to the isolated atoms or molecules. Structural determination of such complexes has relied a lot on indirect methods, such as comparison of the results of various spectroscopies with quantum chemistry calculations. ${ }^{1}$ More specifically, these works often addressed the vibrational 25 spectrum of the complex under study, be it in the IR or far IR frequency range. Size selectivity and isomer selection in these experiments were achieved by combining excitation with an IR and a UV photon. ${ }^{2}$ As a prerequisite for the application of any such method, the complex to be studied must have IR 30 active modes of vibration, and in the case of two-color experiments, contain molecules with a suitable chromophore.

${ }^{a}$ Max-Planck-Institute for Plasma Physics, EURATOM Association, Boltzmannstr. 2, 85748 Garching, Germany. Mailing Address: IPP, c/o BESSY mbH, Albert-Einstein-Str. 15, 12489 Berlin, Germany. Fax: +4930-6392 2990; E-mail: uwe.hergenhahn@ipp.mpg.de

${ }^{b}$ Present Address: BESSY mbH, Albert-Einstein-Str. 15, 12489 Berlin, Germany

Presented at the Bunsen Discussion on Structure and Dynamics of Free Clusters and Nanoparticles using Short Wavelength Radiation, Bad Honnef, 7-9 September 2005.
For systems which lack these prerequisites, much less about their structure is known. On van der Waals clusters, experiments using electron spectroscopy, ${ }^{3,4}$ surface scattering 35 with subsequent mass spectrometry, ${ }^{5}$ and electron diffraction ${ }^{6}$ have been carried out. Simulated results show far more detail than currently is experimentally accessible. ${ }^{7,8}$

In this article we introduce a method that directly identifies the two atomic or molecular species forming an interface in a 40 weakly bonded complex. As an example we use van der Waals clusters of Ar, which are partially covered by a single layer of Ne atoms. We observe a signal easily detectable by electron spectroscopy, which only is present due to the fact that a weak bond between $\mathrm{Ne}$ and $\mathrm{Ar}$ atoms was formed in the cluster 45 condensation process, and which is specific for the two kinds of atoms forming the interface. It occurs as the result of a recently discovered electronic deexcitation process of innervalence vacancies in the Ne layer, which involves an energy transfer to a neighboring site. This process, now termed 50 Interatomic/Intermolecular Coulombic Decay (ICD), is predicted to be generally present in weakly bonded systems of lighter atoms and molecules.

Electronic relaxation processes, such as Auger decay and analysis of X-Ray Fluorescence, have been used to determine 55 elemental compositions of matter for a long time. The first step in the application of any such technique, which is 
creation of a deep core hole, does not assume any special property of the target being probed. Since these decay processes, however, are not sensitive to the chemical neigh60 borhood of the site which is probed, they are not suited for the investigation of the subtle changes in energy and structure brought about by non-covalent complex formation. In contrast, the ICD process potentially depends very sensitively on the environment of the initially excited site. It proceeds as 65 follows: After creation of a vacancy in an inner valence orbital, e.g. by photoionization with VUV radiation, electronic energy is released by filling the vacancy from an outer valence shell. This energy is transferred to a neighboring site, where in turn ionization of an outer valence electron occurs.

${ }_{70}$ Emission of an electron by Auger decay, on the other hand, is a local process, which occurs from the site that was initially ionized. After having been predicted on theoretical grounds, ${ }^{9}$ the existence of ICD and the graphical description outlined above were recently verified experimentally for free van der 75 Waals clusters of Ne. ${ }^{10-12}$

Contrary to intuition ICD does not require a chemical bond between the two sites involved in the energy transfer. Its existence was first inferred from a consideration of the energy levels of doubly charged states in weakly bonded systems. ${ }_{80}$ Compared to the constituent monomers, the double ionization potential in an extended system is always lower, since in states with two positive charges distributed to two different sites their Coulomb repulsion is significantly smaller compared to a doubly charged monomer. This potentially renders 85 inner valence vacancy states unstable against autoionization, which could only decay via fluorescence otherwise. ${ }^{9}$ Consequences of this simple fact were, however, not investigated until recently, since it is not self-evident that an efficient transition mechanism, which directly links a singly ionized to 90 a delocalized doubly ionized state, is viable. Example systems, for which explicit calculations have meanwhile been presented, are $2 \mathrm{~s}^{-1}$ states in $\left(\mathrm{H}_{2} \mathrm{O}\right)_{\mathrm{x}},(\mathrm{HF})_{\mathrm{x}}$ (Ref. 9), $\mathrm{Ne}_{2-13}$ (Ref. 13) and NeAr (Ref. 14). In a recent investigation of the decay mechanism ICD was interpreted as an exchange of a 95 virtual photon in the limit of long bond lengths (weak bonds), this picture being modified by an increasing role of orbital overlap for shorter bonds. ${ }^{15}$ The ICD rate was shown to depend on the bond length $R$ by an $R^{-6}$ law in the long $R$ limit. In this respect, ICD resembles the well-known Förster 100 Resonance Energy Transfer, ${ }^{16}$ but contrary to the latter it can occur by involving atomic subunits of the investigated system and energy conservation is not limiting the possible combinations of 'donor' and 'acceptor' sites. It is therefore a phenomenon of much greater generality.

105 Eventually, the system is left in a state with two opposing positive charges. A weakly bonded dimer will then disintegrate because of the Coulomb repulsion, and this signature of ICD was directly observed in $\mathrm{Ne}_{2} \cdot{ }^{11}$ From the conservation of energy, the total kinetic energy of the ions and the ICD

110 electron has to match the energy difference between the inner valence ionized and the asymptotic, doubly charged final state.

The sensitivity of ICD to the environment of the ionized site was not investigated so far yet, since all experiments on ${ }_{115}$ ICD reported so far were carried out on homogenous clusters. ${ }^{10-12}$ From the above, however, it is clear that ICD involves a simultaneous change of the electronic structure at two different sites, as the result of which one electron will be emitted into the continuum. A sketch of the ICD process in 120 heterogeneous clusters is shown in Fig. 1. Obviously the energy of the secondary electron emitted in this process depends on the energies of inner and outer valence orbitals at the initial site of excitation and at its nearest neighbors.

Here, we report on the ICD signal from a coexpansion of ${ }_{125} \mathrm{Ne}$ and Ar gas into a free molecular beam, by which van der Waals clusters are formed. Determining the composition of these clusters is intricate, and usually requires molecular dynamics simulations for the given expansion parameters., In our work, we will demonstrate the appearance of an ICD 130 signal due to the transition

$\left(\mathrm{Ne} 2 \mathrm{~s}^{-1}\right) \mathrm{Ar} \rightarrow\left(\mathrm{Ne} 2 \mathrm{p}^{-1}\right)\left(\mathrm{Ar} 3 \mathrm{p}^{-1}\right)+\mathrm{e}_{\text {ICD }}^{-}$

which is uniquely related to the formation of a weak bond between $\mathrm{Ne}$ and Ar atoms and which thus signals expansion conditions suitable for the formation of heteroclusters. ${ }_{135}$ Additional insight underpinning this interpretation comes from an analysis of the photoelectron spectra, which are well known for both pure Ne and Ar clusters. ${ }^{12,17}$

\section{Experimental}

The experimental set-up has been described earlier. ${ }^{18}$ Here we 140 report only an outline and some details that have changed with respect to these references. Clusters were formed by supersonic expansion of a gas mixture of Ne and $\operatorname{Ar}$ (99.999\% purity) through a small opening in a copper nozzle. The data displayed in this article were measured using a conical nozzle 145 with a smallest diameter of $80 \mu \mathrm{m}$, a cone length of $1100 \mu \mathrm{m}$ and a full opening angle of $30^{\circ}$. To achieve clustering of inert gases, the nozzle can be cooled down to liquid He temperatures. Gases expanded into a first chamber that is separated from the main experimental chamber by a conical skimmer. 150 The main vacuum chamber was pumped by a cryopump of $900 \mathrm{l} / \mathrm{s}$ capacity mounted opposite to the inlet of the cluster beam and a smaller turbo pump of $360 \mathrm{l} / \mathrm{s}$. Photons were produced by the UE52-SGM undulator beamline of the BESSY synchrotron radiation source (Berlin, Germany). The 155 photon energy resolution amounted to approx. $85 \mathrm{meV}$. Electrons were detected in a commercial hemispherical analyser (Scienta ES 200). Linearly polarized synchrotron radiation was used, and the analyser was mounted at an angle of $54.7^{\circ}$ with respect to the electric field vector, and 160 perpendicular to the beam propagation direction. We have set the pass energy of the electron analyzer to $20 \mathrm{eV}$, and the analyzer resolution to approx. $134 \mathrm{meV}$. For the experiments on clusters with less Ar content, which are mentioned in the discussion but not displayed, a copper nozzle with a short 165 conical section of $100 \mu \mathrm{m}$ diameter and a full opening angle of $30^{\circ}$ was used, and the measurements were done at the U125/1-PGM and U125/2-SGM beamlines of BESSY. Here, typical settings for the photon bandwidth and the analyzer resolution were $100 \mathrm{meV}$ and $80 \mathrm{meV}$, resp. The electron 170 energy analyzer was used at a pass energy of $5 \mathrm{eV}$.

\section{Results}

2 | [journal], [year], [vol], 00-00 
Photoexcited electron spectra from NeAr clusters recorded at a photon energy sufficient for ionization of the Ne $2 \mathrm{~s}$ orbital are shown in Fig. 2. The Figure shows spectra taken at two 175 different temperatures of the nozzle used for the gas expansion (top and bottom row of panels). The ICD transition energy can roughly be calculated from the known atomic ionization potentials involved and the Coulomb repulsion for two positive charges separated by the distance of the $\mathrm{NeAr}$ 180 van der Waals bond. This simple estimate results in a kinetic energy of $7 \mathrm{eV},{ }^{\ddagger}$ same as a more elaborate quantummechanical calculation for the NeAr dimer. ${ }^{20}$ Inspecting the leftmost panels in Fig. 2 we see a peak at about the predicted energy, which is present only at the lower of two expansion 185 temperatures chosen. ICD into a $\left(\mathrm{Ne}^{+}\right)_{2}$ final state, which is also possible in principle, is expected at a much smaller kinetic energy, since the ionization energy of the Ne outer valence level is several eV larger than for the respective level in Ar. In experiments on pure Ne clusters, ICD was observed 190 at around $1.6 \mathrm{eV}$ kinetic energy. ${ }^{10}$ As argued below, we suppose that in our gas beam, at the expansion conditions characterized by this lower temperature, cold Ar clusters covered with a single layer of Ne atoms form. In contrast to that, at the higher of two temperatures only the Ar atoms may 195 condense, such that we have Ar clusters seeded in an atomic Ne beam. Thus, the ICD signal can be used as a monitor for formation of weak NeAr bonds.

A more careful inspection of Fig. 2 shows a second, weaker structure at a kinetic energy about $1.2 \mathrm{eV}$ higher than the main 200 mixed ICD peak. This is in beautiful agreement with simulations of Scheit et al. on mixed ICD in the NeAr dimer that include the final state nuclear dynamics. ${ }^{20}$ There, the higher kinetic energy peak is explained as the combined result of decays from the $v=1$ and $v=2$ vibrationally excited dimer 205 states.

\section{Discussion}

The kinetic energy we observe for the NeAr mixed ICD is larger in our experiment than in the estimates and calculations, because the latter were carried out for a NeAr dimer 210 while in the experiment we probe larger clusters. In the latter, a larger part of the excitation energy is available for the ICD electron because the Coulomb explosion in the final state is hindered by the surrounding atoms, thus less energy is transferred into kinetic energy release of the ionic fragments.

215 The formation of mixed NeAr clusters at an expansion temperature of $66 \mathrm{~K}$ is also plausible from a consideration of the binding energies of van der Waals dimers. For NeAr, the binding energy is $5 \mathrm{meV}$, corresponding to a temperature of $58 \mathrm{~K}^{14}$ Thus, at $66 \mathrm{~K}$ a sufficient fraction of atoms is slow 220 enough to form bonds with the Ar clusters, which act as nuclei for the condensation process. In contrast, the Ne-Ne bond with $3.7 \mathrm{meV}$ binding energy (43 K) is weaker, such that bonding of the Ne to other Ne atoms is unlikely.

$\mathrm{Ne}$ and $\mathrm{Ar}$ photoelectron spectra taken in the same 225 experiment corroborate our inference on the cluster structure. At the higher of two temperatures, the Ar 3s spectrum shows two distinct peaks corresponding to bulk and surface sites of Ar clusters, as can be seen from their binding energy. ${ }^{17} \mathrm{At}$ higher binding energy the atomic 3s peak from a small amount 230 of uncondensed gas is visible. In the region of binding energies pertaining to $\mathrm{Ne} 2 \mathrm{~s}$ photoionization, only a signal from atomic species is visible. In contrast to that, at the lower expansion temperature, the distinction between the two components of the Ar peak is less clear, and in the Ne binding 235 energy range a new peak appears, the binding energy of which is in-between the values for Ne 2 s surface and bulk sites. ${ }^{12}$ Both of these changes can be explained by the formation of a third peak corresponding to NeAr interface sites, which has in a similar manner been observed in ArXe clusters. ${ }^{4}$

240 We can infer further information on the composition of the clusters we probe from the photoelectron spectra. A comparison of the area of $\mathrm{Ne} 2 \mathrm{~s}$ and $\mathrm{Ar} 3 \mathrm{~s}$ photoelectron lines, normalized by the respective cross sections, ${ }^{21}$ gives an approximate measure of the relative Ar content, and for the 245 pure Ar clusters at the higher expansion temperature the bulk/surface ratio gives an approximate measure of the cluster size. ${ }^{22}$ For the spectrum shown in Figure 2, these analyses give an Ar fraction of 0.48(5) and a mean Ar cluster size of $100(20)$ atoms. It should be noted that the clusters exhibit a 250 size distribution ranging from the monomer to values above the mean size. A simple numerical simulation by relaxing 108 $\mathrm{Ne}$ atoms from random positions atop of a core of $100 \mathrm{Ar}$ atoms, the structure of which was taken from the Cambridge Cluster Database, ${ }^{23}$ gives an Ar core, which is densely but not 255 completely covered by a single layer of $\mathrm{Ne}$ atoms. It can be seen that all $\mathrm{Ne}$ atoms have more $\mathrm{Ar}$ than $\mathrm{Ne}$ nearest neighbors. That is, although ICD to states with two Ne 2p vacancies, as seen in earlier work, ${ }^{10-12}$ may compete with the mixed IC decay demonstrated in this article, the latter will be 260 the dominating relaxation mode.

Recent adiabatic simulations of the lowest energy isomers of mixed A, B clusters resulted in inverted structures, that is in our case in Ne clusters covered with $\mathrm{Ar}^{24}$ In contrast to the conditions in our experiment, identical Lennard-Jones 265 parameters for both species A and B were used for these calculations, as only atom size effects, independent of energetic effects, were of interest. We think that the formation of such structures in a coexpansion of the two gases, when the mixture is taken to a low temperature in a short time, is rather 270 unprobable. The formation process of the clusters could be taken into account in a molecular dynamics simulation, but to our knowledge for mixed van-der-Waals-clusters formed in a coexpansion no such simulations are available. However, for mixed cluster formation by pick-up simulations have been 275 carried out, ${ }^{25,26}$ and for pick-up of $\mathrm{Kr}$ atoms by Ar clusters evidence has been presented that the simulated structure is similar to one that was experimentally obtained by coexpansion. ${ }^{25}$ There, different than in Ref. 24 a homogeneous distribution of the $\mathrm{Kr}$ atoms in a $\mathrm{Ar}_{880} \mathrm{Kr}_{120}$ cluster was found. 280 For the pick-up of Ne atoms by Ar clusters these simulations support our idea of an Ar core with Ne layers around it. Only immediately after the time of pick-up, Ne might temporarily penetrate into the Ar core on a ps timescale. ${ }^{26}$ The dimer binding energies (NeNe: $3.7 \mathrm{meV}^{27}$, NeAr: $5 \mathrm{meV}^{14}$, ArAr: $28510.5 \mathrm{meV}^{28}$ ) illustrate this behavior. In an experimental study of the cluster composition for a coexpansion of nitrogen and 
argon, seeding of nitrogen condensation by Ar cores was found as well. ${ }^{29}$

In a series of additional experiments we have probed clusters 290 with a lower fraction of $\mathrm{Ar}$ atoms. The Ar photoelectron signal in these experiments was less intense and neither the $\mathrm{Ne}$ nor the Ar photoelectron signals were showing a clear distinction between bulk and surface sites. The ICD signal appeared at the same kinetic energy as shown in figure 2. Its 295 intensity relative to the $\mathrm{Ne} 2 \mathrm{~s}$ photoelectron line was reproducible for repeated experiments with the same $\mathrm{Ne} / \mathrm{Ar}$ ratio of the clusters, and stayed constant or increased with increasing Ar content. It can therefore be expected that the strength of the ICD signal, in comparison to the initial inner

300 valence signal, can be used not only as a qualitative but also a quantitative monitor for the elemental surroundings of the ionized site.

In bulk metal oxides, recently a non-local resonant electron emission process was discovered and termed MARPE (Multi305 atom Resonant Photoemission). ${ }^{30,31}$ Although the strength of MARPE is now established to be smaller than expected in first experiments, ${ }^{30}$ its existence doubtlessly has been demonstrated. $^{31}$ In comparison to ICD, the same type of quantum mechanical matrix elements is responsible for this 310 effect. However, MARPE is a resonant scattering process involving inner shells and transition energies of several hundred $\mathrm{eV}$, which compete with strong local relaxation channels. The similarity to ICD is therefore mainly formal.

\section{Conclusions}

315 The Interatomic/Intermolecular Coulombic Decay demonstrated in this article should be prevalent for inner-valence vacancy states in weakly bonded systems. It can be understood as a relaxation within the electronic structure, which is mediated to the surroundings of the decaying site.

320 Since information on nearest neighbors is thus imprinted into the electron energy spectra resulting from the decay, and since the vacancy creation and decay processes are entirely general, we expect this process to readily find applications in the analysis of clusters, large, hydrogen-bridge bonded molecules, 325 and solutes.

We would like to thank L.S. Cederbaum, S. Scheit, and V. Averbukh for many stimulating discussions. A. Lindblad participated in some of the measurements in early stages of 330 this project. Partial funding by the Deutsche Forschungsgemeinschaft is gratefully acknowledged.

\section{Notes and references}

$¥$ Ne 2 s atomic binding energy: $48.5 \mathrm{eV}$, Ne $2 \mathrm{p}$ outer valence binding energy: $21.65 \mathrm{eV}$ (average of $2 \mathrm{p}_{1 / 2}, 2 \mathrm{p}_{3 / 2}$ ), Ar $3 \mathrm{p}$ outer valence binding 335 energy: $15.8 \mathrm{eV}$ (average of $3 \mathrm{p}_{1 / 2}, 3 \mathrm{p}_{3 / 2}$ ), final state internuclear distance: $3.5 \AA .{ }^{14,19}$

1 K. Müller-Dethlefs and P. Hobza, Chem. Rev., 2000, 100, 143; U. Buck and F. Huisken, Chem. Rev., 2000, 100, 3863; C. Desfrancois, S. Carles, and J. P. Schermann, Chem. Rev., 2000, 100, 3943; F. N. Keutsch and R. J. Saykally, PNAS, 2001, 98, 10533.

2 E. Nir, K. Kleinermanns, and M. S. de Vries, Nature, 2000, 408, 949; W. Chin, J.-P. Dognon, C. Canuel, F. Piuzzi, I. Dimicoli, M. Mons, I. Compagnon, G. van Helden, and G. Meijer, J. Chem. Phys., 2005, 122, 054317.
3453 E. Rühl, A. P. Hitchcock, P. Morin, and M. Lavollée, Journal de Chimie Physique et de Physico-Chimie Biologique, 1995, 92, 521.

4 M. Tchaplyguine, M. Lundwall, M. Gisselbrecht, G. Öhrwall, R. Feifel, S. Sorensen, S. Svensson, N. Mårtensson, and O. Björneholm, Phys. Rev. A, 2004, 69, 031201.

3505 E. Fort, H. Vach, M. Châtelet, A. D. Martino, and F. Pradére, Eur. Phys. J. D, 2001, 14, 71.

6 G. Torchet, M.-F. de Feraudy, and Y. Loreaux, J. Mol. Struct., 1999, 485-486, 261.

7 D. J. Wales and H. A. Scheraga, Science, 1999, 285, 1368.

3558 J. W. Hewage and F. G. Amar, J. Chem. Phys., 2003, 119, 9021.

9 L. S. Cederbaum, J. Zobeley, and F. Tarantelli, Phys. Rev. Lett., 1997, 79, 4778.

10 S. Marburger, O. Kugeler, U. Hergenhahn, and T. Möller, Phys. Rev. Lett., 2003, 90, 203401.

36011 T. Jahnke, A. Czasch, M. S. Schöffler, S. Schössler, A. Knapp, M. Käsz, J. Titze, C. Wimmer, K. Kreidi, R. E. Grisenti, A. Staudte, O. Jagutzki, U. Hergenhahn, H. Schmidt-Böcking, and R. Dörner, Phys. Rev. Lett., 2004, 93, 163401.

12 G. Öhrwall, M. Tchaplyguine, M. Lundwall, R. Feifel, H. Bergersen, T. Rander, A. Lindblad, J. Schulz, S. Peredkov, S. Barth, S. Marburger, U. Hergenhahn, S. Svensson, and O. Björneholm, Phys. Rev. Lett., 2004, 93, 173401.

13 R. Santra, J. Zobeley, and L. S. Cederbaum, Phys. Rev. B, 2001, 64, 245104.

37014 J. Zobeley, R. Santra, and L. S. Cederbaum, J. Chem. Phys., 2001, 115, 5076.

15 V. Averbukh, I. B. Müller, and L. S. Cederbaum, Phys. Rev. Lett., 2004, 93, 263002.

16 G. D. Scholes, Annu. Rev. Phys. Chem., 2003, 54, 57.

37517 R. Feifel, M. Tchaplyguine, G. Öhrwall, M. Salonen, M. Lundwall, R. R. T. Marinho, M. Gisselbrecht, S. L. Sorensen, A. Naves de Brito, L. Karlsson, N. Mårtensson, S. Svensson, and O. Björneholm, Eur. Phys. J. D, 2004, 30, 343.

18 S. P. Marburger, O. Kugeler and U. Hergenhahn, Eighth International 380 Conference on Synchrotron Radiation Instrumentation, San Francisco, 25-29 Aug. 2003, edited by T. Warwick, J. Arthur, H. A. Padmore and J. Stöhr, AIP Conference Proceedings, New York, no.708, 2004, pp. 1114-17.; S. Barth, S. Joshi, S. Marburger, V. Ulrich, A. Lindblad, G. Öhrwall, O. Björneholm, and U. Hergenhahn, J. Chem. Phys., 2005, 122, 241102.

19 G. P. Williams, Electron Binding Energies in X-Ray Data Booklet, 2nd ed.(eds Thompson, A. C. et al.) 1-1 - 1-7 (Lawrence Berkeley National Laboratory, 2001), http://xdb.lbl.gov; S. M. Cybulski and R. R. Toczylowski, J. Chem. Phys., 1999, 111, 10520.

39020 S. Scheit, V. Averbukh, H.-D. Meyer, J. Zobeley, and L.S. Cederbaum, J. Chem. Phys., 2006, 124, 154305.

21 B. Möbus, B. Magel, K.-H. Schartner, B. Langer, U. Becker, M. Wildberger, and H. Schmoranzer, Phys. Rev. A, 1993, 47, 3888; J. M. Bizau and F. J. Wuilleumier, J. Electron Spectrosc. Relat. Phenom., 1995, 71, 205

22 M. Tchaplyguine, R. R. Marinho, M. Gisselbrecht, J. Schulz, N. Mårtensson, S. L. Sorensen, A. Naves de Brito, R. Feifel, G. Öhrwall, M. Lundwall, S. Svensson, and O. Björneholm, J. Chem. Phys., 2004, 120, 345.

40023 The Cambridge Cluster Database, http://www-wales.ch.cam.ac.uk/CCD.html.

24 J. P. K. Doye and L. Meyer, Phys. Rev. Lett., 2005, 95, 063401.

25 E. Fort, H. Vach, M. Châtelet, A. De Martino, and F. Pradère, Eur. Phys. J. D, 2001, 14, 71.

40526 H. Vach, J. Chem. Phys., 1999, 111, 3536.

27 F. Sebastianelli, E. Yurtsever, F.A. Gianturco, Int. J. Mass Spectrom., 2002, 220, 193.

28 E. A. Colbourn and A. E. Douglas, J. Chem. Phys., 1976, 65, 1741.

29 E. Fort, F. Pradère, A. De Martino, H. Vach, and M. Châtelet, Eur. Phys. J. D, 1998, 1, 79.

30 A. Kay, E. Arenholz, S. Mun, F. J. García de Abajo, C. S. Fadley, R. Denecke, Z. Hussain, and M. A. Van Hove, Science, 1998, 281, 679

31 A. W. Kay, F. J. Garcia de Abajo, S.-H. Yang, E. Arenholz, B. S. Mun, N. Mannella, Z. Hussain, M. A. Van Hove, and C. S. Fadley, Phys. Rev. B, 2001, 63, 115119.

4 | [journal], [year], [vol], 00-00 


\section{Single column figure/scheme (below)}

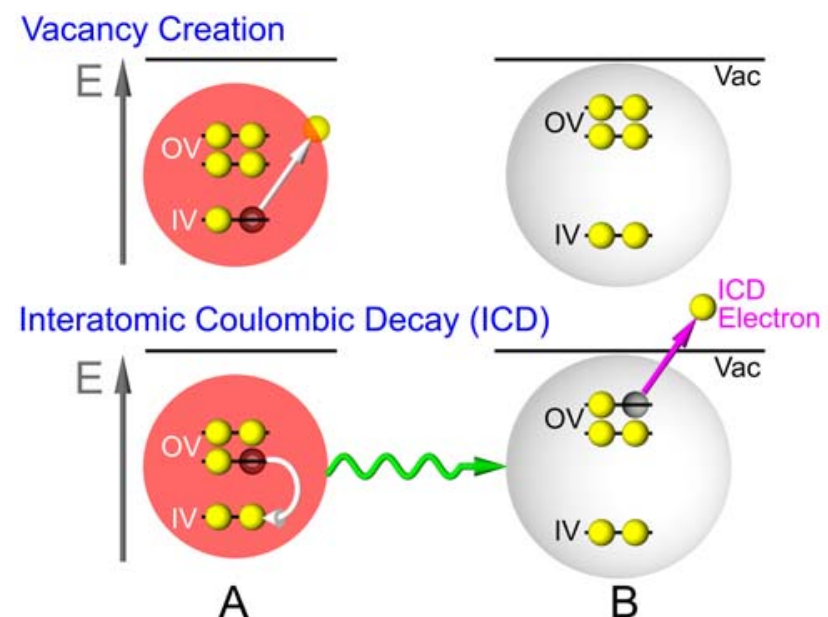

Fig. 1 The Interatomic Coulombic Decay. Creation of a vacancy in an inner-valence level, e.g. by photoionization, disposes energy into a weakly bonded system, here a dimer $\mathrm{AB}$. In the subsequent decay process, the initial vacancy is filled from an outer valence level; energy released by this process is transferred to a neighbouring site $B$, which in turn is ionized. This process typically proceeds on a fs time scale. ${ }^{12,13}$

\section{Single column figure/scheme (below)}

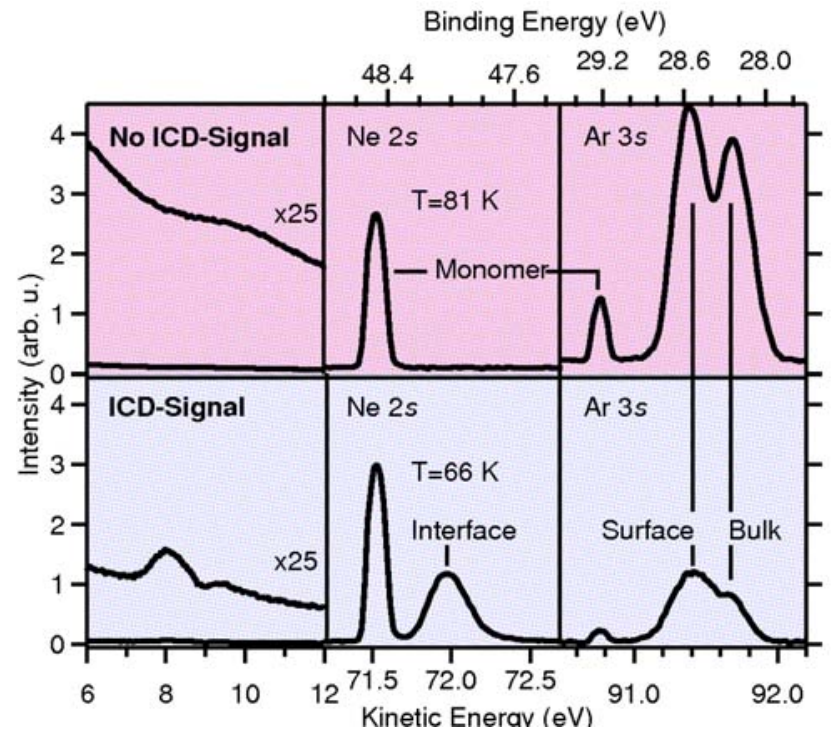

Fig. 2 Spectra of photoelectrons and subsequently emitted ICD electrons from a NeAr coexpansion. In the bottom row of panels, conditions suitable for formation of mixed NeAr clusters were selected. The photoelectron spectra show the presence of Ar bulk, surface and interface sites (right) and of Ne interface sites (middle). Consequentially a signal from ICD can be detected (left). At a higher expansion temperature, Ne atoms cannot condense and the molecular beam consists of pure $\mathrm{Ar}$ clusters and atomic Ne (top row). From Ne, only the monomer photoelectron signal can be seen (middle) and the ICD signal vanishes (left). The photon energy was at $120 \mathrm{eV}$ for both expansion temperatures. The intensity scale is in arbitrary units but the range is the same for all panels. 